\title{
Significance of Cross-Linking
}

\section{of $\alpha_{2}$-Plasmin Inhibitor to Fibrin in Inhibition of}

\author{
Fibrinolysis and in Hemostasis
}

\author{
Yoichi Sakata and Nobuo Aoki, Institute of Hematology and Department of \\ Medicine, Jichi Medical School, Tochigi-Ken 329-04, Japan
}

\begin{abstract}
A B S T R A C T When blood is clotted, $\alpha_{2}$-plasmin inhibitor $\left(\alpha_{2} \mathrm{PI}\right)$ is cross-linked to fibrin by activated fibrin-stabilizing factor (activated coagulation Factor XIII, plasma transglutaminase). The amount of crosslinked $\alpha_{2}$-PI is proportional to the amount of $\alpha_{2}$ PI present at the time of clotting. Plasma from a patient with congenital deficiency of $\alpha_{2}$ PI was supplemented with various amounts of purified $\alpha_{2}$ PI. Clots were prepared from these plasmas and were suspended in plasma containing a normal concentration of $\alpha_{2} \mathrm{PI}$, and spontaneous clot lysis was observed. When the clot was formed in the presence of calcium ions and thereby allowing cross-linking to occur, the rate and extent of fibrinolysis were found to be inversely proportional to the concentrations of $\alpha_{2}$ PI present in the clot at the time of clotting. When the clot was formed in the absence of calcium ions so that no cross-linking occurred, the clot underwent fibrinolysis at similar rates, regardless of the concentrations of $\alpha_{2} \mathrm{PI}$ in the clot. When the clot formed in the presence of calcium ions was squeezed and washed to remove unbound proteins before being suspended in plasma, the extent of fibrinolysis was also inversely proportional to the amount of $\alpha_{2}$ PI cross-linked to fibrin. Similar results were obtained when the clot was suspended in buffered saline instead of plasma. These observations suggest that spontaneous fibrinolysis is mainly carried out by plasminogen/plasminogen activator bound to fibrin, and this fibrinolysis caused by fibrin-associated activation of plasminogen was mainly inhibited by $\alpha_{2}$ PI crosslinked to fibrin. To further support this concept, $\alpha_{2}$ PI treated with activated fibrin-stabilizing factor and that had lost most of its cross-linking capacity was used in similar experiments. This modified $\alpha_{2}$ PI had the same inhibitory activity on plasmin as the native inhibitor, but gave significantly less inhibition of fibrinolysis in
\end{abstract}

Received for publication 9 September 1981 and in revised form 2 November 1981. every experiment, particularly when the clot was compacted by platelet-mediated clot retraction or by squeezing. Thus, it was concluded that $\alpha_{2} \mathrm{PI}$ crosslinked to fibrin plays a significant role in inhibition of physiologically occurring fibrinolysis. It is further suggested that the absence of cross-linked $\alpha_{2}$ PI contributes to accelerated fibrinolysis and hemorrhagic tendency in patients with congenital deficiency of fibrin-stabilizing factor.

\section{INTRODUCTION}

$\alpha_{2}$-Plasmin inhibitor $\left(\alpha_{2} \mathrm{PI}\right)^{1}$ or $\alpha_{2}$-antiplasmin is a glycoprotein of a 63,000-67,000 estimated mol wt, and is one of the serine-proteinase inhibitors existing in plasma (1). Its concentration in normal plasma is $6.9 \pm 0.6 \mathrm{mg} / 100 \mathrm{ml}(\simeq 1 \mu \mathrm{M})(2) . \alpha_{2} \mathrm{PI}$ is produced by liver parenchymal cells (3), and the plasma concentration is reduced in liver diseases (4). $\alpha_{2}$ PI inhibits various serine proteinases including trypsin, chymotrypsin, and proteases participating in blood coagulation, fibrinolysis, and the kinin-forming system (5, 6). Its major role in blood plasma, however, is inhibition of plasmin-catalyzed fibrinolysis (7-11), and its congenital deficiency results in a severe hemorrhagic tendency (12).

The concentration of $\alpha_{2} \mathrm{PI}$ in the circulating blood is readily reduced when plasminogen is activated under thrombolytic therapy or in disseminated intravascular coagulation, because $\alpha_{2}$ PI rapidly inhibits plasmin evolved by forming a complex, and the complex is removed from the circulating blood by the reticuloendothelial system (9-11).

In addition to the rapid inactivation of plasmin, $\alpha_{2} \mathrm{PI}$ interferes with adsorption of plasminogen to fi-

\footnotetext{
${ }^{1}$ Abbreviations used in this paper: FSF, fibrin-stabilizing factor; $\alpha_{2}$ PI, $\alpha_{2}$-plasmin inhibitor; KIU, kallikrein inhibitor unit.
} 
brin, resulting in an efficient inhibition of fibrinolysis (13). Furthermore, $\alpha_{2} \mathrm{PI}$ is cross-linked to fibrin when blood is clotted, thereby making the fibrin clot less susceptible to lysis by plasmin (2). The cross-linking is catalyzed by activated fibrin-stabilizing factor (activated FSF, fibrinoligase, blood coagulation Factor XIIIa, plasma transglutaminase), and the amount of $\alpha_{2} \mathrm{PI}$ cross-linked to fibrin is dependent on the concentrations of activated FSF (FSFa), fibrin, and $\alpha_{2} \mathrm{PI}$ (2). Reduced resistance of a plasma fibrin clot prepared from FSF-deficient plasma to plasmin-catalyzed fibrinolysis may be, at least partially, accounted for by the lack of $\alpha_{2}$ PI cross-linking in FSF-deficient plasma. It is also known that another plasma component called plasma fibronectin is cross-linked to fibrin when blood is clotted (14). The cross-linking is also catalyzed by FSFa. However, cross-linking of $\alpha_{2} \mathrm{PI}$ to fibrin occurs independently of fibronectin cross-linking, and the presence or absence of plasma fibronectin does not influence the rate and extent of $\alpha_{2}$ PI cross-linking to fibrin (15).

The purpose of this paper is to demonstrate the significance of $\alpha_{2}$ PI cross-linking to fibrin in inhibition of fibrinolysis and infer from this information its meaning in hemostasis.

\section{METHODS}

Plasma. Blood was collected from antecubital veins of normal subjects or a patient with congenital deficiency of $\alpha_{2}$ PI (12) into 0.1 vol of $3.8 \%$ sodium citrate, and it was centrifuged at $2,000 \mathrm{~g}$ for $20 \mathrm{~min}$ to prepare platelet-poor plasma. To obtain platelet-rich plasma, the citrated blood was centrifuged at $650 \mathrm{~g}$ for $4 \mathrm{~min}$. Plasma was used for the experiments within $30 \mathrm{~min}$ after drawing the blood. Venipuncture in the patient and in normal subjects was performed after informed consent for the procedure was obtained.

\section{Proteins}

$\alpha_{2} P I . \quad \alpha_{2}$ PI was purified by the previously described method (1). The concentration was determined spectrophotometrically using absorbance $A_{1 \mathrm{~cm}}^{1 \mathrm{~s}}=7.03$ at $280 \mathrm{~nm}$ (1). The protein was dissolved in Tris-buffered saline $(0.05 \mathrm{M}$ Tris$\mathrm{HCl}, 0.15 \mathrm{M} \mathrm{NaCl}, \mathrm{pH}$ 7.4).

Fibrinogen. Human fraction I-4 prepared according to the method of Blombäck and Blombäck (16) was used as the fibrinogen preparation after removing contaminating plasminogen using lysine-Sepharose (17). The concentration was determined spectrophotometrically using $A_{1 \mathrm{~cm}}^{1 \%}=15.1(16)$, and the coagulable protein was $>95 \%$.

Plasminogen. Native glutamyl-plasminogen, whose amino-terminal group is glutamic acid, was prepared from fresh plasma in the presence of aprotinin $(10 \mathrm{KIU} / \mathrm{ml})$ (Mochida Pharm. Comp., Tokyo, Japan) by affinity chromatography with lysine-Sepharose followed by DEAE-Sephadex chromatography (18).

Radioiodination. The fibrinogen and plasminogen preparations were radioiodinated by the solid-state lactoperoxidase method of David (19) using lactoperoxidase (Calbio-
chem-Behring Corp., American Hoechst Corp., San Diego, Calif.) and ${ }^{125} \mathrm{INa}(17 \mathrm{Ci} / \mathrm{ml}$ ) (New England Nuclear, Boston, Mass.). The labeled fibrinogen preparation had a radioactivity of $6.29 \times 10^{4} \mathrm{cpm} / \mu \mathrm{g}$. Coagulability was not changed by iodination, and the fibrinogen concentration of the labeled preparation was $291 \mathrm{mg} / 100 \mathrm{ml}$ clottable protein. The labeled plasminogen preparation had a radioactivity of 2.23 $\times 10^{5} \mathrm{cpm} / \mu \mathrm{g}$ and the concentration was $1.1 \mathrm{mg} / \mathrm{ml}$. After iodination the plasminogen preparation was found to have preserved its form, and no two-chain plasmin form was detected by sodium dodecyl sulfate (SDS) polyacrylamide gel electrophoresis (20).

Thrombin. Purified thrombin was prepared from a bovine thrombin preparation (Parke, Davis \& Co., Detroit, Mich.) according to the method of Lundblad (21), and was stored as a $600 \mathrm{U} / \mathrm{ml}$ solution in $50 \%$ glycerol at $-20^{\circ} \mathrm{C}$.

Hirudin. The hirudin (a thrombin inhibitor obtained from leeches) used was a grade IV preparation from the Sigma Chemical Co., St. Louis, Mo., and was demonstrated to have no plasmin inhibitor activity when tested by the chromogenic (22) and the clot lysis (9) methods.

FSF. Purified FSF was prepared from human plasma by the described method (23). The concentration of purified FSF was determined by measuring the absorbance of the FSF solution at $280 \mathrm{~nm}$ and converting absorbance to protein concentration using an extinction coefficient $\mathrm{E}_{\mathrm{cm}}^{1 \mathrm{x}}=13.8$ for pure FSF (24). FSF activity was assayed by an antibody neutralization method (25) using the clotting Factor XIII test kit supplied by Behringwerke AG, Marburg, Germany. 1 unit of FSF is defined as the amount of FSF present in 1 $\mathrm{ml}$ of normal pooled standard plasma, and the specific activity of the purified FSF was $40 \mathrm{U} / \mathrm{mg}$ protein. Thrombinmodified FSF was prepared by incubating purified FSF ( 2 $\mathrm{mg} / \mathrm{ml})$ with thrombin $(5 \mathrm{U} / \mathrm{ml})$ in Tris-buffered saline at $25^{\circ} \mathrm{C}$ for $30 \mathrm{~min}$. Subsequently the mixture was mixed with $3 \mathrm{mM}$ diisopropyl fluorophosphate (Sigma Chemical Co.) to inactivate thrombin, incubated further for $60 \mathrm{~min}$ at $25^{\circ} \mathrm{C}$, and then dialyzed against $0.05 \mathrm{M}$ Tris- $\mathrm{HCl}$ buffer, $\mathrm{pH} \mathrm{7.4,}$ containing $1 \mathrm{mM} \mathrm{EDTA}$, at $4^{\circ} \mathrm{C}$ for $24 \mathrm{~h}$. The preparation was stable at $4^{\circ} \mathrm{C}$ for at least a week. Thrombin-modified FSF thus obtained was converted to active FSF by calcium ions added at the time of the experiments.

$F S F$-treated $\alpha_{2} P I . \quad \alpha_{2} P I(600 \mu \mathrm{g} / \mathrm{ml})$ was incubated with thrombin-modified FSF $(25 \mu \mathrm{g} / \mathrm{ml})$ and calcium chloride $(5 \mathrm{mM})$ in Tris-buffered saline for $8 \mathrm{~h}$ at $25^{\circ} \mathrm{C}$. The $\alpha_{2} \mathrm{PI}$ thus treated and the original $\alpha_{2}$ PI were separately iodinated with ${ }^{125} \mathrm{I}$, and both were tested for their susceptibility to FSF-catalyzed cross-linking with fibrin as previously described (2). When $2.5 \mathrm{mg} / \mathrm{ml}$ of fibrinogen was used, $34 \%$ of native $\alpha_{2} \mathrm{PI}$ and $5 \%$ of FSF-treated $\alpha_{2} \mathrm{PI}$ were cross-linked to fibrin, indicating that FSF-treated $\alpha_{2} \mathrm{PI}$ had lost most of its susceptibility to the FSF-catalyzed cross-linking reaction and retained only $15 \%$ of the cross-linking capacity of normal $\alpha_{2} \mathrm{PI}$. The other two functional properties other than crosslinking capacity namely, the immediate inhibition of plas$\min (1)$, and the interference with the binding of plasminogen to fibrin $(13,26)$, were totally intact in FSFtreated $\alpha_{2}$ PI.

Plasminogen activation during the spontaneous clot lysis. Plasminogen activation during spontaneous clot lysis was followed by measuring amidolytic activity generated and by observing the distribution of radioactivity in SDS gel electrophoresis of the labeled plasminogen added to plasma before clotting. 100- $\mu$ l aliquots of $\alpha_{2} \mathrm{PI}$-deficient fresh plateletpoor plasma or normal plasma was mixed with $3 \mu \mathrm{l}$ of the radiolabeled plasminogen, $3 \mu$ l of calcium chloride $(0.05 \mathrm{M})$, and $5 \mu \mathrm{l}$ of thrombin $(40 \mathrm{U} / \mathrm{ml})$. The clot formed was in- 
cubated at $37^{\circ} \mathrm{C}$. For amidolytic assay, $192 \mu \mathrm{l}$ of Tris-buffered saline and $700 \mu \mathrm{l}$ of $3 \mathrm{mM}$ H-D-valyl-leucyl-lysinyl-pnitroaniline (a chromogenic substrate for plasmin, S-2251) (Kabi Diagnostica, Stockholm, Sweden) were added to the clot after various lengths of time. The clot was immediately squeezed with a bamboo stick to express as much fluid as possible from the clot. The mixture containing the suspended clot was further incubated at $37^{\circ} \mathrm{C}$. $3 \mathrm{~min}$ after the addition of $\mathrm{S}-2251,100 \mu \mathrm{l}$ of $50 \%$ acetic acid was added and mixed to stop the reaction. Absorbance of the liquid phase at $\mathbf{4 0 5}$ $\mathrm{nm}$ was measured, and the amidolytic activity on S-2251 was expressed as nanokatals per milliliter of original mixture using an absorbance value of 10.5 at $405 \mathrm{~nm}$ for $1 \mathrm{mM}$ nitroaniline. As a blank, acetic acid was added to the clot at the time of addition of S-2251. For the observation of distribution of radioactivity in SDS gel electrophoresis, 900 $\mu \mathrm{l}$ of $10 \mathrm{M}$ urea/10\% SDS/10\% 2-mercaptoethanol solution were added to the clot after various lengths of time and immediately heated in a boiling water for $30 \mathrm{~min}$ to obtain the solubilized sample. The sample was mixed with $25 \mu \mathrm{l}$ of $50 \%$ glycerol and then subjected to SDS polyacrylamide gel electrophoresis on $5 \%$ polyacrylamide gel (20). After fixing with $50 \%$ methanol $/ 10 \%$ acetic acid mixture, the gel was cut into slices of 1-mm thickness using gel slicer HP 100 (Hotta Rika, Tokyo). Each sliced gel was counted for radioactivity. Plasminogen activation was also assessed by comparing plasminogen activity in plasma before clotting with that in serum after complete lysis of the clot. Plasminogen activity was assayed by the caseinolytic method (27).

Spontaneous in vitro fibrinolysis. $5 \mathrm{ml}$ of $\alpha_{2}$ PI-deficient fresh platelet-poor plasma was mixed with $10 \mu$ l of radiolabeled fibrinogen. $200 \mu$ l of the mixture was mixed with 10 $\mu$ l of $\alpha_{2}$ PI of various concentrations or buffered saline. Each of these mixtures was clotted with $10 \mu \mathrm{l}$ of thrombin $(50 \mathrm{U} /$ $\mathrm{ml}$ ) containing $0.5 \mathrm{M}$ calcium chloride or $0.06 \mathrm{M}$ EDTA. After incubation at $37^{\circ} \mathrm{C}$ for $30 \mathrm{~min}$, the clot formed was suspended in $1 \mathrm{ml}$ of fresh normal plasma containing 2.5 mM EDTA and 1 unit of hirudin, and was further incubated at $37^{\circ} \mathrm{C}$ for as long as $24 \mathrm{~h}$. The purposes of including EDTA and hirudin in the suspending plasma milieu were to prevent the suspending plasma from being clotted by thrombin or gelated by activated FSF released from the clot. In some experiments, the clot was first squeezed with a bamboo stick against the wall of the tube to express as much fluid as possible, washed three times by soaking it in $2 \mathrm{ml}$ of Tris-buffered saline containing $2 \%$ of albumin for $5 \mathrm{~min}$ at room temperature each time, and then finally suspended in $1 \mathrm{ml}$ of plasma or buffered saline containing $2 \%$ albumin. To measure fibrinolysis, $25-\mu \mathrm{l}$ aliquots of the suspending plasma or buffered saline were removed at intervals for counting of radioactivity. Results were expressed as the percent release of radiolabel, which was calculated from the counts, applying a correction for the influence of repeated subsampling upon the volume of the supernatant. In other experiments, the clot formed was simply left in the tube without being suspended in plasma. After various lengths of incubation, the clot was squeezed and $25 \mu \mathrm{l}$ of the liquid phase was counted for radioactivity. Radioactivity was counted using the Auto-Well gamma system, Aloka ARC-451 (Aloka Co., Tokyo, Japan).

\section{RESULTS}

Plasminogen activation during spontaneous clot lysis. When the $\alpha_{2}$ PI-deficient fresh plasma clot was incubated at $37^{\circ} \mathrm{C}$, the clot was spontaneously lysed after several hours. Plasmin generation during the spontaneous clot lysis was followed by assaying for amidolytic activity and by SDS gel electrophoresis using radiolabeled plasminogen. There was a small amidolytic activity towards $\mathrm{S}-2251$ in plasma before clotting, and this activity was not increased for the lst $h$ of incubation after clotting (Fig. 1). During this period of time, the peak of radioactivity was not changed and there was no appearance of a new peak of radioactivity in SDS gel electrophoresis. After this lag time, there was an appearance of a new distinct peak of radioactivity with a concomitant rise of amidolytic activity. The new peak of radioactivity corresponded to the light chain of plasmin. The peak of the heavy chain was partly buried in the slope of the large peak of plasminogen. Any other peak suggestive of the formation of plasmin-inhibitor complex was not detected. The height of the peak of the light chain and amidolytic activity rapidly increased and reached plateau levels after an $\sim 8$-h incubation, when the clot was completely lysed (Fig. 1).

Plasminogen activities of the plasma samples before clotting were $2.4 \pm 0.15 \mathrm{U} / \mathrm{ml}(n=5)$, whereas those of the serum samples (clotted and lysed samples) were $2.15 \pm 0.07 \mathrm{U} / \mathrm{ml}(n=5)$. The difference was significant $(P<0.02)$ and $\sim 10 \%$ of the average plasminogen activity of the plasma samples. Since plasminogen concentration in the plasma was $\sim 2 \mu \mathrm{M}$ (12), plasmin generated during the clot lysis was calculated to be $\sim 0.2 \mu \mathrm{M}$.

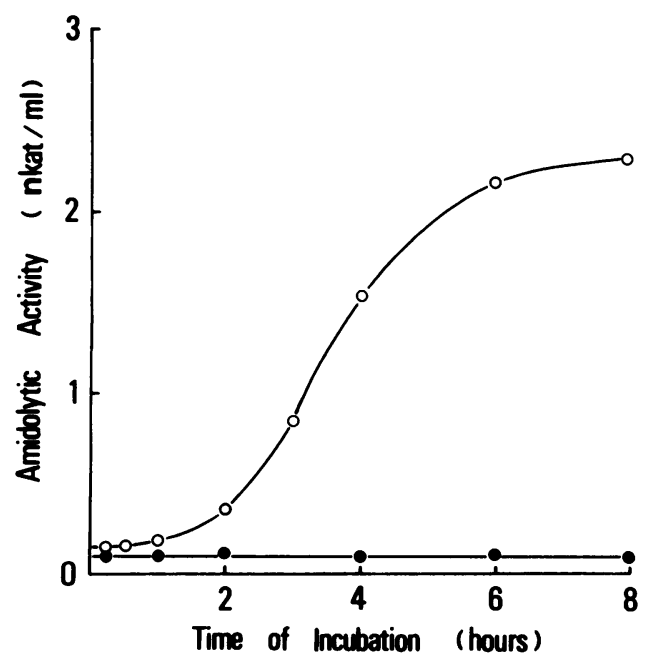

FIgURE 1 Development of amidolytic activity during spontaneous clot lysis. $\alpha_{2}$ PI-deficient citrated plasma $(O)$ or normal plasma (O) was clotted by thrombin in the presence of calcium ions, and incubated at $37^{\circ} \mathrm{C}$. After various lengths of time, the amidolytic activity developed was measured using S-2251 as a substrate. nkat, nanomoles of substrate hydrolyzed per second. (Methods). 
When normal plasma was clotted and incubated, no fibrinolysis was observed and there was neither appearance of the light chain nor rise of amidolytic activity (Fig. 1) because any plasmin generated would have been immediately neutralized by $\alpha_{2} \mathrm{PI}$.

Spontaneous fibrinolysis of the clot suspended in plasma or buffered saline. When the fresh plasma clots containing various subnormal concentrations of $\alpha_{2}$ PI were suspended in fresh plasma, remarkable fibrinolysis occurred in spite of the presence of a normal concentration of $\alpha_{2} \mathrm{PI}$ in the suspending plasma milieu. The rate of fibrinolysis, however, was dependent on whether calcium ions were present or not at the time of clotting. When calcium ions were present, FSF-catalyzed cross-linking occurred, and the rate of fibrinolysis was inversely related to the concentration of $\alpha_{2} \mathrm{PI}$ present at the time of clotting (Fig. 2). When calcium ions were absent, FSF-catalyzed cross-linking did not occur and the rate of fibrinolysis was accelerated. Furthermore, the rate of fibrinolysis became less dependent on the $\alpha_{2}$ PI concentration in the clotting plasma (Fig. 2).

To determine if the dependence of the lysis rate of cross-linked fibrin on $\alpha_{2} \mathrm{PI}$ concentration in the clotting plasma was due to the difference in the amount of $\alpha_{2}$ PI bound to fibrin, the clot formed in the presence of calcium ions was squeezed and washed to remove proteins unbound to fibrin before suspending the clot in plasma. The results obtained were similar to those of cross-linked fibrin in Fig. 2 and demonstrated again that fibrinolysis was inhibited in proportion to the $\alpha_{2} \mathrm{PI}$ concentration in the clotting plasma at the time of clotting. Omission of the washing procedure (the clot was only squeezed before being suspended in plasma) did not appreciably affect the results.

When the clots formed in the presence of calcium ions were squeezed, washed, and suspended in buffered saline instead of plasma, fibrinolysis was still observed; and inhibition of fibrinolysis was similarly dependent on the $\alpha_{2}$ PI concentration in the clotting plasma (Fig. 3).

Effects of FSF-treated $\alpha_{2} P I$ on fibrinolysis. To further determine the effect of cross-linking of $\alpha_{2} \mathrm{PI}$ to fibrin on the lysis rate, FSF-treated $\alpha_{2}$ PI that had lost nearly $85 \%$ of its cross-linking capacity but fully maintained all the other functions was used and compared with the native $\alpha_{2} \mathrm{PI}$ in the same experiments as those described above in the presence of calcium ions. FSF-treated $\alpha_{2}$ PI gave less inhibition of fibrinolysis under both experimental conditions, with and without washing of the clot. When the clot was not washed, the dependence of inhibition of fibrinolysis on $\alpha_{2}$ PI concentration in the clotting plasma was still seen with FSF-treated $\alpha_{2}$ PI, although inhibition of fibrinolysis became less. When the clot was squeezed

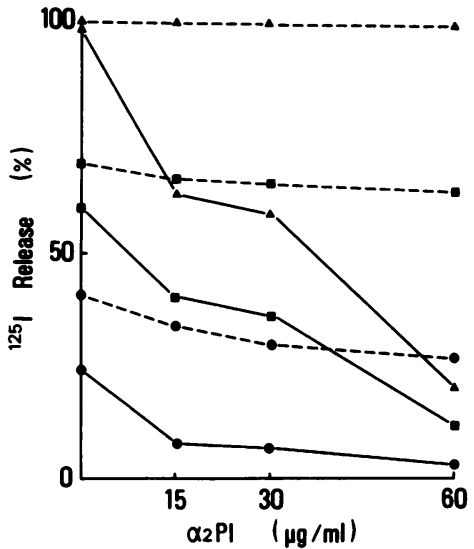

Figure 2 Spontaneous in vitro fibrinolysis of plasma clots suspended in plasma. Citrated platelet-poor plasma containing radiolabeled fibrinogen and various amounts of $\alpha_{2} \mathrm{PI}$ was clotted by thrombin in the presence (solid line) or the absence (broken line) of calcium ions. The clot formed was suspended in normal plasma and incubated at $37^{\circ} \mathrm{C}$. At 2 $(O), 6(\square)$, and $24(\Delta)$ h of incubation, release of radiolabel from the clot into the suspending plasma milieu was measured and expressed as a percentage of the total radioactivity. The concentrations of $\alpha_{2} \mathrm{PI}$ in the clot at the time of clotting are given on the abscissa. (Methods).

and washed, FSF-treated $\alpha_{2}$ PI gave markedly less inhibition of fibrinolysis; and the difference of inhibition as a result of the different amount of $\alpha_{2} \mathrm{PI}$ present in the clotting plasma became very small and was seen only during the early period of fibrinolysis (Fig. 4).

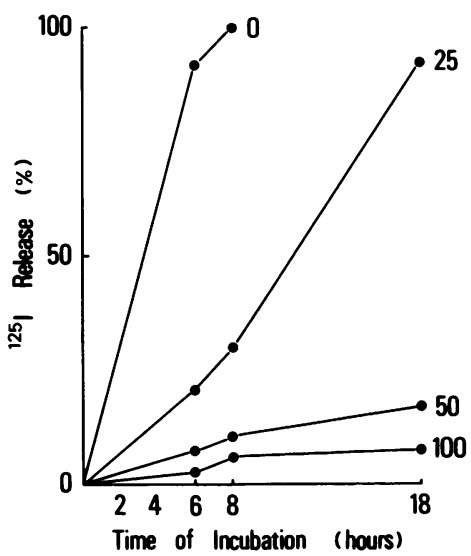

Figure 3 Time-course of spontaneous in vitro fibrinolysis of washed clots suspended in buffered saline. The experimental procedures were the same as those in Fig. 2 except that the plasma clot was squeezed, washed to remove unbound proteins, and suspended in buffered saline instead of plasma. All the plasma clots were formed in the presence of calcium ions. The concentrations of $\alpha_{2} \mathrm{PI}$ in the clots at the time of clotting are given as a percentage of normal concentration in citrated plasma $(60 \mu \mathrm{g} / \mathrm{ml})$ at the ends of the curves. (Methods). 


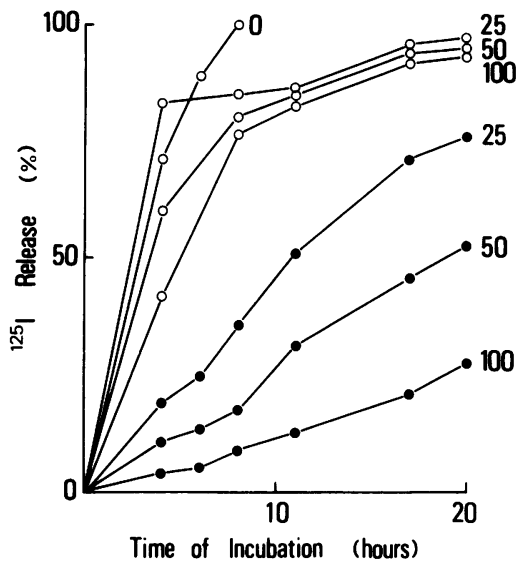

Figure 4 Comparison between native and FSF-treated $\alpha_{2}$ PI in their influences on spontaneous in vitro fibrinolysis of washed clots suspended in plasma. Citrated platelet-poor plasma containing radiolabeled fibrinogen and various amounts of native (O) or FSF-treated (O) $\alpha_{2}$ PI was clotted in the presence of calcium ions. The clot formed was squeezed, washed, and suspended in normal plasma, and fibrinolysis was observed as in Fig. 2. The concentrations of $\alpha_{2} \mathrm{PI}$ in the clots at the time of clotting are given as a percentage of the normal concentration in citrated plasma (60 $\mu \mathrm{g} / \mathrm{ml}$ ) at the ends of the curves. (Methods).

Platelet-rich or poor plasma, containing $50 \%$ of the normal concentration of native or FSF-treated $\alpha_{2} \mathrm{PI}$, was clotted in the presence of calcium ions, and incubated at $37^{\circ} \mathrm{C}$. The platelet-rich plasma clot was retracted maximally within $2 \mathrm{~h}$. When native $\alpha_{2}$ PI was used, platelet-rich plasma clot underwent only slightly more accelerated fibrinolysis than the platelet-poor plasma clot (Fig. 5). When FSF-treated $\alpha_{2}$ PI was used, however, the difference became pronounced and remarkably enhanced fibrinolysis was observed with the platelet-rich plasma clot (Fig. 5).

\section{DISCUSSION}

When $\alpha_{2}$ PI-deficient plasma obtained from a patient with congenital deficiency of $\alpha_{2} \mathrm{PI}(12)$ was clotted and incubated at $37^{\circ} \mathrm{C}$, the clot underwent spontaneous lysis, and complete lysis was observed after several hours of incubation $(12,28)$. In this study, even when the clot was squeezed, washed to remove unbound protein, and suspended in buffered saline, the clot still underwent spontaneous lysis. This indicates that spontaneous fibrinolysis was carried out by plasminogen/ plasminogen activators bound to fibrin during the clot formation.

When the clot was suspended in normal plasma containing a normal concentration of $\alpha_{2} \mathrm{PI}$, the clot still underwent spontaneous lysis. Clot lysis was complete at 8-24 h of incubation in spite of a sufficient amount of $\alpha_{2} \mathrm{PI}(\simeq 1 \mu \mathrm{M})$ in the suspending plasma milieu to neutralize plasmin generated $(\simeq 0.2 \mu \mathrm{M})$ during clot lysis. Since no appreciable generation of amidolytic activity and no formation of plasmin light chain that contains active site were observed during the lst $h$ of clot incubation (Fig. 1), plasmin responsible for lysis of the clot must have been generated in the clot while the clot was being suspended in the plasma milieu, but not during the initial $30 \mathrm{~min}$ alloted for clot formation before suspension in plasma. Thus, fibrinolysis carried out by plasmin generated while the clot was being suspended in plasma was not appreciably affected by $\alpha_{2} \mathrm{PI}$ present in the surrounding plasma milieu.

When $\alpha_{2}$ PI-deficient plasma was supplemented with various amounts of $\alpha_{2} \mathrm{PI}$ and then clotted in the presence of calcium ions, fibrinolysis was inhibited in proportion to the amounts of $\alpha_{2}$ PI present in the clotting plasma (Figs. 2 and 3). Since the amount of $\alpha_{2} \mathrm{PI}$ crosslinked to fibrin is directly proportional to the concentrations of $\alpha_{2}$ PI present in the clot at the time of clotting $(2,15)$, it can be said that inhibition of fibrinolysis is dependent on the amounts of $\alpha_{2}$ PI cross-linked to fibrin. The cross-linking reaction of $\alpha_{2}$ PI to fibrin is very rapid and complete within a few minutes at $37^{\circ} \mathrm{C}$ (15). Therefore, the period of $30 \mathrm{~min}$ alloted for clot formation in the present studies was considered to be long enough to allow the maximum cross-linking of $\alpha_{2} \mathrm{PI}$, in addition to the complete $\gamma$-cross-linking and the partial $\alpha$-cross-linking of fibrin.

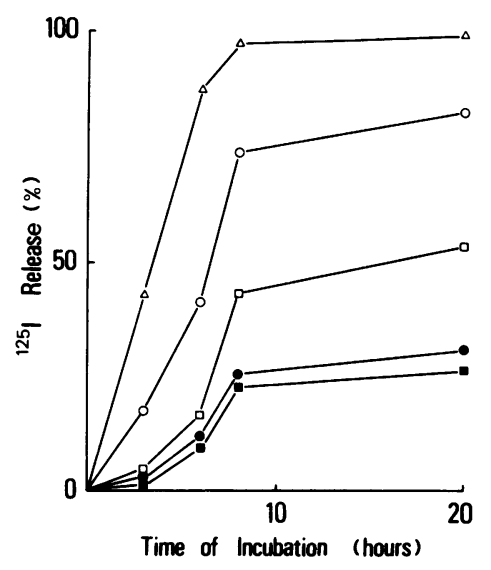

FIGURE 5 Spontaneous clot lysis. $200 \mu \mathrm{l}$ of platelet-rich $\alpha_{2}$ PI-deficient plasma or platelet-poor $\alpha_{2}$ PI-deficient plasma containing radioiodinated fibrinogen was mixed with $10 \mu \mathrm{l}$ of $0.6 \mathrm{mg} / \mathrm{ml}$ native or FSF-treated $\alpha_{2}$ PI to give $\sim 50 \%$ of normal concentration of $\alpha_{2} \mathrm{PI}$ in citrated plasma, and clotted in the presence of calcium ions. The radioactivity released into the liquid phase was counted after various lengths of time, and expressed as a percentage of the total original counts. Platelet-rich plasma supplemented with FSF-treated (O) or native (O) $\alpha_{2}$ PI. Platelet-poor plasma supplemented with FSF-treated $(\square)$ or native $(\square) \alpha_{2}$ PI. Platelet-rich plasma without supplement of $\alpha_{2}$ PI $(\Delta)$. 
In the absence of calcium ions, so that no cross-linking can occur, fibrinolysis was accelerated and $\alpha_{2} \mathrm{PI}$ dependent inhibition of fibrinolysis became virtually ineffective (Fig. 2).

From these results, it may be suggested that spontaneous fibrinolysis that is the result of the fibrin-associated process of plasminogen activation is mainly inhibited by $\alpha_{2}$ PI cross-linked to fibrin. If this is true, $\alpha_{2} \mathrm{PI}$, which is only devoid of the cross-linking capacity but maintains all its other functions, should give considerably less inhibition of fibrinolysis. To examine this possibility, FSF-treated $\alpha_{2}$ PI was used. Treatment of the protein with activated FSF causes an irreversible hydrolysis of the $\gamma$-carboxamide group of the glutamine residue susceptible to the action of activated FSF (29), resulting in a loss of $\sim 85 \%$ of the cross-linking capacity of $\alpha_{2}$ PI. When this FSF-treated $\alpha_{2}$ PI was used in the experiments, a markedly less inhibition of fibrinolysis was observed, particularly when the squeezed clots were suspended in plasma (Fig. 4).

In in vivo thrombus formation, platelets are involved and clot retraction takes place. Since clot retraction has a similar effect produced by squeezing the clot, the present studies using a squeezed clot may be regarded as a simulation of clot retraction. Therefore, platelet-rich $\alpha_{2}$ PI-deficient plasma supplemented with native or FSF-treated $\alpha_{2}$ PI was clotted in the presence of calcium ions, and its spontaneous fibrinolysis was compared with that obtained with platelet-poor plasma. The results indicated that decreases in inhibition of fibrinolysis with FSF-treated $\alpha_{2} \mathrm{PI}$ was more pronounced in the platelet-rich plasma clot than that in the platelet-poor plasma clot (Fig. 5). In other words, inhibitory activity of FSF-treated $\alpha_{2}$ PI on fibrinolysis was minimized when clot retraction took place, because the most of FSF-treated $\alpha_{2}$ PI was not bound to fibrin and squeezed out from the clot when the clot was retracted. This emphasizes that cross-linked $\alpha_{2} \mathrm{PI}$ plays more important role in inhibition of spontaneous lysis of retracted clot than in inhibition of spontaneous lysis of nonretracted clot.

It is interesting to note that the amount of crosslinked $\alpha_{2} \mathrm{PI}$ is $\sim 0.2 \mu \mathrm{mol} /$ liter plasma when normal plasma is clotted (2), and this amount is nearly equivalent to the amount of plasmin generated during the spontaneous lysis of an $\alpha_{2}$ PI-deficient plasma clot. Because of this, usually no fibrinolysis is observed when a normal plasma clot is incubated.

All these experimental results suggest that spontaneous fibrinolysis is mainly caused by fibrin-associated plasminogen activation (activation of fibrin-bound plasminogen by fibrin-bound plasminogen activator), and effectively inhibited by $\alpha_{2} \mathrm{PI}$ cross-linked to fibrin. The absence of $\alpha_{2}$ PI cross-linking to fibrin (2) may largely contribute to an accelerated fibrinolysis of he- mostatic plugs and bring about hemorrhagic diathesis in patients with congenital deficiency of FSF.

\section{ACKNOWLEDGMENTS}

We are grateful to Dr. M. Kohakura for allowing us to obtain blood from a patient with congenital deficiency of $\alpha_{2}$ PI. We gratefully acknowledge the cooperation of Dr. T. Tamaki and the technical assistance of Ms. K. Tateno.

This work was supported in part by a research grant for cardiovascular diseases (55A-1) from the Ministry of Health and Welfare, and a research grant from the Ministry of Education, Japan.

\section{REFERENCES}

1. Moroi, M., and N. Aoki. 1976. Isolation and characterization of $\alpha_{2}$-plasmin inhibitor from human plasma. A novel proteinase inhibitor which inhibits activator-induced clot lysis. J. Biol. Chem. 251: 5956-5965.

2. Sakata, Y., and N. Aoki. 1980. Cross-linking of $\alpha_{2}$-plasmin inhibitor to fibrin by fibrin-stabilizing factor. $J$. Clin. Invest. 65: 290-297.

3. Aoki, N. 1979. $\alpha_{2}$-Plasmin inhibitor. A newly discovered protease inhibitor in human plasma. In The Microembolism Syndrome. T. Saldeen, editor. Almqvist och Wiksell International, Stockholm. 123-151.

4. Aoki, N., and T. Yamanaka. 1978. The $\alpha_{2}$-plasmin inhibitor levels in liver diseases. Clin. Chim. Acta. 84: 99105.

5. Moroi, M., and N. Aoki. 1977. Inhibition of proteases in coagulation, kinin-forming, and complement systems by $\alpha_{2}$-plasmin inhibitor. J. Biochem. (Tokyo). 82: 969-972.

6. Saito, H., G. H. Goldsmith, M. Moroi, and N. Aoki. 1979. The inhibitory spectrum of $\alpha_{2}$-plasmin inhibitor. Proc. Natl. Acad. Sci. U. S. A. 76: 2013-2017.

7. Müllertz, S., and I. Clemmensen. 1976. The primary inhibitor of plasmin in human plasma. Biochem. J. 159: 545-553.

8. Collen, D. 1976. Identification and some properties of a new fast-reacting plasmin inhibitor in human plasma. Eur. J. Biochem. 69: 209-216.

9. Aoki, N., M. Moroi, M. Matsuda, and K. Tachiya. 1977. The behavior of $\alpha_{2}$-plasmin inhibitor in fibrinolytic states. J. Clin. Invest. 60: 361-369.

10. Collen, D., and B. Wiman. 1978. Fast-acting plasmin inhibitor in human plasma. Blood. 51: 563-569.

11. Aoki, N. 1979. Natural inhibitors of fibrinolysis. 1979. Prog. Cardiovasc. Dis. 21: 267-286.

12. Aoki, N., H. Saito, T. Kamiya, K. Koie, Y. Sakata, and M. Kobakura. 1979. Congenital deficiency of $\alpha_{2}$-plasmin inhibitor associated with severe hemorrhagic tendency. J. Clin. Invest. 63: 877-884.

13. Aoki, N., M. Moroi, and K. Tachiya. 1978. Effects of $\alpha_{2}$-plasmin inhibitor on fibrin clot lysis. Its comparison with $\alpha_{2}$-macroglobulin. Thromb. Haemostasis. 39: 2231.

14. Mosher, D. F. 1975. Cross-linking of cold-insoluble globulin by fibrin-stabilizing factor. J. Biol. Chem. 250: 6614-6621.

15. Tamaki, T., and N. Aoki. 1981. Cross-linking of $\alpha_{2}$-plasmin inhibitor and fibronectin to fibrin by fibrin-stabilizing factor. Biochim. Biophys. Acta. 661: 280-286.

16. Blombäck, B., and M. Blombäck. 1956. Purification of human and bovine fibrinogen. Ark. Kemi. 10: 415-443.

17. Matsuda, M., S. Iwanaga, and S. Nakamura. 1972. A 
simple, large scale method for preparation of plasminogen-free fibrinogen. Thromb. Res. 1: 619-630.

18. Wallen, P., and B. Wiman. 1972. Characterization of human plasminogen. Separation and partial chàracterization of different molecular forms of human plasminogen. Biochim. Biophys. Acta. 257: 122-134.

19. David, G. S. 1972. Solid state lactoperoxidase: a highly stable enzyme for simple, gentle, iodination of proteins. Biochem. Biophys. Res. Commun. 48: 464-471.

20. Weber, K., and M. Osborn. 1969. The reliability of molecular weight determinations of dodecyl sulfate-polyacrylamide gel electrophoresis. J. Biol. Chem. 244: 4406-4412.

21. Lundblad, R. L. 1971. A rapid method for the purification of bovine thrombin and the inhibition of the purified enzyme with phenyl methyl sulfonyl fluoridide. Biochemistry. 10: 2501-2505.

22. Naito, K., and N. Aoki. 1977. Assay of $\alpha_{2}$-plasmin inhibitor activity by means of a plasmin specific tripeptide substrate. Thromb. Res. 12: 1147-1156.
23. Curtis, C. G., and L. Lorand. 1976. Fibrin-stabilizing factor (Factor XIII). Methods Enzymol. 45: 177-191.

24. Schwartz, M. L., S. V. Pizzo, R. L. Hill, and P. A. McKee. 1973. Human Factor XIII from plasma and platelets. $J$. Biol. Chem. 248: 1395-1407.

25. Bohn, H., and J. Haupt. 1968. Eine quantitative Bestimmung von Faktor XIII mit Anti-Faktor XIII Serum. Thromb. Diath. Haemorrh. 19: 309-315.

26. Aoki, N., and Y. Sakata. 1980. Influence of $\alpha_{2}$-plasmin inhibitor on adsorption of plasminogen to fibrin. Thromb. Res. 19: 149-155.

27. Aoki, N., M. Moroi, Y. Sakata, N. Yoshida, and M. Matsuda. 1978. Abnormal plasminogen. A hereditary molecular abnormality found in a patient with recurrent thrombosis. J. Clin. Invest. 61: 1186-1195.

28. Aoki, N., Y. Sakata, M. Matsuda, and K. Tateno. 1980. Fibrinolytic states in a patient with congenital deficiency of $\alpha_{2}$-plasmin inhibitor. Blood. 55: 483-488.

29. Chung, S. I., and J. E. Folk. 1972. Kinetic studies with transglutaminases. J. Biol. Chem. 247: 2798-2807. 